\title{
CORRIGENDUM
}

\section{Associations of markers in 11 obesity candidate genes with maximal weight loss and weight regain in the SOS bariatric surgery cases}

\author{
MA Sarzynski ${ }^{1}$, P Jacobson ${ }^{2}$, T Rankinen ${ }^{1}$, B Carlsson ${ }^{2}$, L Sjöström ${ }^{2}$, C Bouchard ${ }^{1}$ and LMS Carlsson ${ }^{2}$ \\ ${ }^{1}$ Human Genomics Laboratory, Pennington Biomedical Research Center, Baton Rouge, LA, USA and ${ }^{2}$ The Institutes of Medicine, \\ Sahlgrenska Academy, Gothenburg University, Gothenburg, Sweden
}

International Journal of Obesity (2012) 36, 1016; doi:10.1038/ijo.2012.81

Correction to: International Journal of Obesity (2011) 35, 676-683; doi:10.1038/ijo.2010.166

Sarzynski et al. ${ }^{1}$ reported the association of obesity candidate genes with weight loss and regain after bariatric surgery in the May 2011 issue of Int J Obesity. Unfortunately, 30 subjects were affected by errors that occurred during plating of DNA samples.

We have re-analyzed the data after excluding the aforementioned subjects. Overall, our findings and interpretation remain unchanged. In the re-analysis, after taking into account the multiple testing corrected threshold for significance of $P<0.00033$, the only statistically significant association with maximal weight loss was still found at FTO SNP rs16945088, and the $P$-value for this association did not change from the original report $(P=0.0002)$. Furthermore, in the re-analysis none of the
SNPs were significantly associated with weight regain after taking into account multiple testing, which is the same finding as the original report.

Thus, our overall interpretation of the results does not change even after excluding the 30 subjects from the analyses.

The authors would like to apologise for their error.

\section{REFERENCE}

1 Sarzynski MA, Jacobson P, Rankinen T, Carlsson B, Sjöström L, Bouchard C et al. Associations of markers in 11 obesity candidate genes with maximal weight loss and weight regain in the SOS bariatric surgery cases. Int J Obes 2011; 35 676-683. 